\title{
Mises à jour du Guide canadien d'immunisation : avril 2015 à octobre 2016
}

\author{
Jensen $\mathrm{C}^{1}$, Lerch $\mathrm{R}^{1}$ au nom du Comité consultatif national de l'immunisation (CCNI)*
}

\section{Résumé}

Le Guide canadien d'immunisation ( $\mathrm{GCl}$ ) est un résumé fiable et facile à lire de renseignements et de recommandations sur l'immunisation dont les fournisseurs de soins de santé et les responsables des politiques se servent depuis des décennies. II est constamment mis à jour en fonction des nouvelles recommandations du Comité consultatif national de l'immunisation (CCNI) et du Comité consultatif de la médecine tropicale et de la médecine des voyages (CCMTMV), deux organismes consultatifs externes de l'Agence de la santé publique du Canada. En septembre 2016, le $\mathrm{GCl}$ a adopté une nouvelle plateforme Web, dont la navigabilité a été améliorée, de même que la version mobile, plus conviviale. Entre avril 2015 et octobre 2016, cinq nouvelles déclarations du CCNI ont été publiées et reflétées dans le GCl. L'objectif de cet article est de proposer au lecteur les faits saillants des principaux changements récemment apportés aux recommandations concernant les vaccins actifs dans le $\mathrm{GCl}$. Par exemple, pour ce qui est du vaccin contre l'hépatite $A(H A)$, il peut désormais être administré à partir de l'âge de six mois. Son administration peut également être envisagée pour tous les sujets recevant, à plusieurs reprises, des facteurs de coagulation dérivés du plasma. De nouvelles recommandations ont également été formulées pour l'utilisation des immunoglobulines pour la prophylaxie post-exposition. Dans le cas du vaccin contre le virus du papillome humain (VPH), tous les vaccins autorisés contre le VPH au Canada, y compris le vaccin VPH9, peuvent être utilisés conformément aux calendriers d'immunisation recommandés contre le VPH. Concernant le vaccin antigrippal, les adultes présentant des troubles neurologiques ou du développement neurologique ont été ajoutés dans le groupe auquel la vaccination antigrippale est particulièrement recommandée. L'utilisation du vaccin antigrippal à forte dose a été approuvée au Canada pour les adultes de 65 ans et plus. En outre, le vaccin antigrippal vivant atténué (VVAl) n'est plus un produit recommandé de préférence chez les enfants et les adolescents. Le vaccin conjugué contre le pneumocoque 13-valent (PNEU-C-13) peut être recommandé, au cas par cas, aux adultes immunocompétents de 65 ans et plus qui n'ont pas déjà été immunisés contre les pneumococcies. Lorsqu'il est administré, il doit précéder le vaccin polysaccharidique 23-valent contre le pneumocoque (PNEU-P-23). Les immunoglobulines contre la varicelle peuvent désormais être administrées jusqu'à 10 jours après la dernière exposition afin d'atténuer la maladie. Un certain nombre de modifications mineures ont été apportées aux critères d'évaluation de l'immunité contre la varicelle.

\section{Affiliation}

${ }^{1}$ Centre de l'immunisation et des maladies respiratoires infectieuses, Agence de la santé publique du Canada, Ottawa (Ontario)

*Correspondance : naci-ccni@ phac-aspc.gc.ca

Citation proposée : Jensen C, Lerch R au nom du Comité consultatif national de l'immunisation (CCNI). Mises à jour du Guide canadien d'immunisation : avril 2015 à octobre 2016. Relevé des maladies transmissibles au Canada 2016;42(12):285-8. https://doi.org/10.14745/ccdr.v42i12a04f

\section{Introduction}

Depuis 1979, le Guide canadien d'immunisation (GCl) constitue un résumé fiable et facile à lire de renseignements sur l'immunisation pour les fournisseurs de soins de santé qui administrent des vaccins à leurs patients et pour les responsables des politiques pour la prestation de programmes d'immunisation. Le GCl, publié par l'Agence de la santé publique du Canada (ASPC), traduit en une seule et unique ressource les recommandations et les conseils du Comité consultatif national de l'immunisation (CCNI) et du Comité consultatif de la médecine tropicale et de la médecine des voyages (CCMTMV). Le CCNI est un organisme consultatif de I'ASPC qui formule des recommandations sur l'utilisation des vaccins actuellement ou récemment approuvés pour l'administration chez l'humain au Canada, notamment en signalant les groupes à risque qui devraient être la cible des campagnes de vaccination pour les maladies évitables par la vaccination (1). Le CCMTMV est un organisme consultatif composé d'experts qui aide l'Agence à formuler des conseils en matière de santé des voyageurs pour les professionnels de la santé. Les recommandations du CCNI et du CCMTMV sont publiées par l'ASPC et résumées dans le $\mathrm{GCl}$ (2).

Le $\mathrm{GCl}$ comporte cinq parties, qui traitent des renseignements clés sur l'immunisation, de la sécurité des vaccins, des populations spéciales, des vaccins actifs et des agents d'immunisation passive. La partie 4, qui aborde les vaccins actifs, est organisée en différents chapitres, tous propres à une maladie, et présente des renseignements sur l'épidémiologie et 
les caractéristiques d'une maladie, ainsi que des renseignements et des recommandations d'utilisation propres à chaque vaccin. II s'agit de la partie du $\mathrm{GCl}$ la plus souvent mise à jour en fonction des nouvelles recommandations.

Le $\mathrm{GCl}$ est mis à jour par le CCNI. Les chapitres sont mis à jour à mesure que de nouvelles données probantes sur les vaccins et les maladies évitables par la vaccination sont accessibles, et à mesure que des déclarations et mises à jour du CCNI et du CCMTMV sont publiées. Depuis 2012, le GCl est publié en ligne dans un format électronique (2). Un tableau des mises à jour résume les principaux changements apportés aux différents chapitres. La date du dernier examen ou de la dernière mise à jour du chapitre est notée sur la page Web respective. En septembre 2016, le $\mathrm{GCl}$ a adopté une nouvelle plateforme Web et possède désormais une version mobile conviviale; autant d'améliorations qui se traduisent, pour l'utilisateur, par une plus grande navigabilité.

L'objectif de cet article est de proposer au lecteur les faits saillants des principaux changements apportés aux recommandations concernant les vaccins actifs dans le
$\mathrm{GCl}$ depuis sa dernière mise à jour, à savoir d'avril 2015 à octobre 2016 (3).

\section{Approche}

Durant l'élaboration de ses recommandations, le CCNI effectue des synthèses approfondies de connaissances et des analyses englobant des examens scientifiques, des pratiques en évolution et des recommandations à l'échelle nationale et internationale. Les recommandations sont ensuite résumées dans les chapitres correspondants du $\mathrm{GCl}$. Des recommandations détaillées concernant l'immunisation et l'utilisation des vaccins offerts au Canada se trouvent dans les déclarations et les mises à jour des déclarations (1).

\section{Résumé des mises à jour}

Le tableau 1 récapitule les changements récents et les renseignements ajoutés au $\mathrm{GCl}$. Les recommandations obsolètes y sont également signalées.

Tableau 1 : Faits saillants des principaux changements apportés aux recommandations concernant les vaccins actifs dans le Guide canadien d'immunisation d'avril 2015 à octobre 2016

\begin{tabular}{|c|c|c|}
\hline Vaccin actif & Recommandations précédentes & Nouvelles recommandations \\
\hline \multirow[t]{4}{*}{$\begin{array}{l}\text { Hépatite A } \\
\text { (HA) }\end{array}$} & $\begin{array}{l}\text { Le vaccin contre l'hépatite A (HA) peut être administré aux sujets } \\
\text { âgés de douze mois et plus. }\end{array}$ & $\begin{array}{l}\text { Le vaccin contre l'hépatite } A(H A) \text { peut être administré aux sujets âgés de six mois et plus } \\
\text { (4). }\end{array}$ \\
\hline & $\begin{array}{l}\text { L'immunisation contre HA peut être envisagée pour les personnes } \\
\text { souffrant d'hémophilie } A \text { ou B qui reçoivent des facteurs de } \\
\text { coagulation dérivés du plasma. }\end{array}$ & $\begin{array}{l}\text { L'immunisation avec le vaccin contre HA peut être envisagée pour tous les sujets recevant, } \\
\text { à plusieurs reprises, des facteurs de coagulation dérivés du plasma (4). }\end{array}$ \\
\hline & Aucune recommandation précédente. & $\begin{array}{l}\text { Pour la prophylaxie post-exposition dans les } 14 \text { jours suivant l'exposition d'adultes réceptifs } \\
\text { de } 60 \text { ans et plus qui sont des contacts familiaux ou étroits d'un cas, les immunoglobulines } \\
\text { humaines ordinaires (lg) peuvent être administrées en plus du vaccin contre HA (4). }\end{array}$ \\
\hline & $\begin{array}{l}\text { L'immunisation contre HA est recommandée pour tous les sujets } \\
\text { réceptifs non immunisés atteints d'hépatopathie chronique, } \\
\text { notamment ceux infectés par le virus de l'hépatite } C \text { et les } \\
\text { porteurs de l'hépatite } B \text { chronique, car la maladie pourrait être } \\
\text { plus grave dans leur cas. }\end{array}$ & $\begin{array}{l}\text { Pour la prophylaxie post-exposition de sujets réceptifs souffrant d'une hépatopathie } \\
\text { chronique, les immunoglobulines doivent être administrées dans les } 14 \text { jours suivant } \\
\text { l'exposition, en plus du vaccin contre HA (4). }\end{array}$ \\
\hline $\begin{array}{l}\text { Virus du } \\
\text { papillome } \\
\text { humain (VPH) }\end{array}$ & $\begin{array}{l}\text { Vaccination avec le vaccin VPH2 ou VPH4, conformément aux } \\
\text { calendriers recommandés d'immunisation contre le VPH. }\end{array}$ & $\begin{array}{l}\text { L'ensemble des vaccins contre le VPH actuellement autorisés au Canada, y compris le } \\
\text { vaccin VPH9 (Gardasi }{ }^{\circledR} 9 \text { ) récemment autorisé, peuvent être utilisés conformément aux } \\
\text { calendriers recommandés d'immunisation contre le VPH (5). }\end{array}$ \\
\hline \multirow[t]{5}{*}{ Grippe } & $\begin{array}{l}\text { Le vaccin antigrippal n'était indiqué que pour les enfants atteints } \\
\text { de troubles neurologiques ou du développement neurologique. }\end{array}$ & $\begin{array}{l}\text { Les adultes souffrant de troubles neurologiques ou du développement neurologique ont } \\
\text { été ajoutés au groupe auquel la vaccination antigrippale est particulièrement recommandée } \\
\text { (6). }\end{array}$ \\
\hline & Aucune recommandation précédente. & $\begin{array}{l}\text { L'administration du vaccin antigrippal Fluzone }{ }^{\circledR} \text { à forte dose a été approuvée au Canada } \\
\text { chez les adultes âgés de } 65 \text { ans et plus (6). }\end{array}$ \\
\hline & & $\begin{array}{l}\text { Des données probantes permettent d'affirmer que le vaccin antigrippal trivalent inactivé à } \\
\text { forte dose devrait offrir aux personnes âgées une protection supérieure à celle des vaccins } \\
\text { par voie intramusculaire à dose standard. }\end{array}$ \\
\hline & $\begin{array}{l}\text { Le vaccin antigrippal vivant atténué (VVI) était un produit } \\
\text { recommandé de préférence pour les enfants et les adolescents } \\
\text { âgés de } 2 \text { à } 17 \text { ans. }\end{array}$ & $\begin{array}{l}\text { Le vaccin antigrippal vivant atténué (VVAl) n'est plus un produit recommandé de préférence } \\
\text { pour les enfants et les adolescents âgés de } 2 \text { à } 17 \text { ans (8). }\end{array}$ \\
\hline & $\begin{array}{l}\text { Il n'existe actuellement aucune donnée démontrant que } \\
\text { l'administration du WAl aux personnes allergiques aux œufs } \\
\text { est sans danger, cette pratique n'est donc actuellement pas } \\
\text { recommandée. }\end{array}$ & Les personnes allergiques aux œufs peuvent être vaccinées avec le VVAI (8). \\
\hline Pneumocoque & $\begin{array}{l}\text { Aucune recommandation précédente concernant l'administration } \\
\text { du vaccin PNEU-C-13 aux adultes immunocompétents de } 65 \text { ans } \\
\text { et plus. }\end{array}$ & $\begin{array}{l}\text { Le vaccin conjugué contre le pneumocoque } 13 \text {-valent (PNEU-C-13) peut être recommandé, } \\
\text { au cas par cas, aux adultes immunocompétents de } 65 \text { ans et plus qui n'ont pas déjà été } \\
\text { immunisés contre les pneumococcies, pour la prévention de la pneumonie d'origine } \\
\text { communautaire (CAP) et des pneumococcies invasives (PI) causées par les } 13 \text { sérotypes de } \\
\text { pneumocoque contenus dans ce vaccin conjugué. Lorsqu'il est administré, il doit précéder } \\
\text { le vaccin polysaccharidique } 23 \text {-valent contre le pneumocoque (PNEU-P-23) (9). }\end{array}$ \\
\hline \multirow[t]{2}{*}{ Varicelle } & $\begin{array}{l}\text { Les recommandations précédentes relatives aux intervalles } \\
\text { minimaux concernaient des produits spécifiques. }\end{array}$ & $\begin{array}{l}\text { Bien que l'intervalle recommandé entre deux vaccins à composant antivaricelleux soit } \\
\text { toujours d'au moins trois mois pour les enfants de moins de } 13 \text { ans et de six semaines pour } \\
\text { les sujets de } 13 \text { ans et plus, un intervalle de quatre semaines peut être envisagé dans des } \\
\text { circonstances exceptionnelles. Si la deuxième dose de vaccin à composant antivaricelleux } \\
\text { est administrée à un intervalle inférieur à quatre semaines, elle doit être répétée (10). }\end{array}$ \\
\hline & $\begin{array}{l}\text { Les effets bénéfiques des immunoglobulines contre la varicelle } \\
\text { (Varlg) sont maximaux si elles sont administrées dans les } 96 \text { heures } \\
\text { suivant la première exposition. Si plus de } 96 \text { heures se sont } \\
\text { écoulées depuis la dernière exposition, les effets bénéfiques de } \\
\text { l'administration de Varlg sont incertains. }\end{array}$ & $\begin{array}{l}\text { Les immunoglobulines contre la varicelle peuvent être administrées jusqu'à dix jours après } \\
\text { la dernière exposition afin d'atténuer la maladie (11). }\end{array}$ \\
\hline
\end{tabular}


Outre les modifications identifiées dans le tableau concernant le vaccin contre la varicelle, d'autres précisions ont été apportées aux critères d'évaluation de la réceptivité relative à l'immunité contre la varicelle, à l'utilisation des antécédents auto-déclarés et au diagnostic des fournisseurs de soins de santé, ainsi qu'aux recommandations à l'intention des travailleurs de la santé, des femmes enceintes et des sujets immunodéprimés (12).

\section{Conclusion}

Le $\mathrm{GCl}$ est continuellement mis à jour afin de présenter les nouvelles données scientifiques et les nouvelles pratiques, comme le reflètent les déclarations et les mises à jour les plus récentes des déclarations du CCNI et du CCMTMV. L'ASPC s'engage à fournir des renseignements concernant I'immunisation et les vaccins offerts au Canada dans un format facilement accessible et lisible grâce à des mises à jour opportunes et suivies du $\mathrm{GCl}$.

Vous pouvez poser vos questions ou nous faire part de vos commentaires relatifs au $\mathrm{GCl}$, aux déclarations ou aux revues de littérature par le biais de la fonction Contactez-nous au CCNI (13).

Pour obtenir des renseignements concernant les nouvelles recommandations et déclarations du $\mathrm{CCNI}$, ainsi que sur les mises à jour et/ou mises à jour des chapitres du $\mathrm{GCl}$, veuillez vous abonner à la liste de diffusion du CCNI (14).

\section{Remerciements}

Les auteurs tiennent à remercier les membres extrêmement dévoués du CCNI, ainsi que le personnel de I'ASPC pour leur soutien dans le cadre du processus de mise à jour du $\mathrm{GCl}$.

\section{Conflit d'intérêts}

Aucun.

\section{Financement}

L'ASPC soutient les activités du CCNI en tant qu'organisme consultatif externe.

\section{Références}

1. Comité consultatif national de l'immunisation (CCNI) [Internet]. Recommandations, déclarations supplémentaires et mises à jour. Ottawa: ASPC; 2016 [Mise à jour le 17 oct 2016; consulté le 12 oct 2016]. Disponible à l'adresse : http://www.phac-aspc.gc.ca/naci-ccni/index-fra.php\#rec.

2. Comité consultatif national de l'immunisation (CCNI). Guide canadien d'immunisation [Internet]. Ottawa: PHAC;
2016 [consulté le 12 oct 2016]. Disponible à l'adresse : http://healthycanadians.gc.ca/healthy-living-vie-saine/ immunization-immunisation/canadian-immunization-guidecanadien-immunisation/index-fra.php.

3. Gemmill I, Quach $\mathrm{C}$ au nom du Comité consultatif national de l'immunisation (CCNI). Mises à jour apportées au Guide canadien d'immunisation: De mars 2014 à mars 2015 [Internet]. Relevé des dies transmissibles au Canada. 20 avr 2015;41(S3). Disponible à l'adresse : http://www.phac-aspc. gc.ca/publicat/ccdr-rmtc/15vol41/dr-rm41s-3/updates-mj04fra.php.

4. Une déclaration d'un comité consultatif (DCC) Comité consultatif national de l'immunisation (CCNI). Mise à jour des recommandations concernant I'utilisation du vaccine contre l'hépatite A [Internet]. Ottawa: ASPC; 2016 [consulté le 12 oct 2016]. Disponible à l'adresse : http://www. healthycanadians.gc.ca/publications/healthy-living-vie-saine/ hepatitis-a-vaccine-update-recommended-use-2016-mise-ajour-recommandations-hepatite-a-vaccin/index-fra.php.

5. Une déclaration d'un comité consultatif (DCC)

Comité consultatif national de l'immunisation (CCNI). Recommandations mises à jour sur les vaccins contre le virus du papillome humain (VPH) : vaccine nonavalent contre le VPH et précisions sur les intervalles minimums entre les doses dans le calendrier d'immunisation contre le VPH [Internet]. Ottawa: ASPC; 2016 [consulté le 12 oct 2016]. Disponible à l'adresse : http://www.healthycanadians.gc.ca/ publications/healthy-living-vie-saine/human-papillomavirus9-valent-vaccine-update-recommendation-mises-a-jourrecommandations-papillome-humain-vaccin-nonavalent/ index-fra.php?_ga=1.177483553.56611766.1473697342.

6. Une déclaration d'un comité consultatif (DCC) Comité consultatif national de l'immunisation (CCNI). Chapitre sur la grippe du Guide canadien d'immunisation et Déclaration sur la vaccination antigrippale pour la saison 2016-2017 [Internet]. Ottawa: ASPC; 2016 [consulté le 12 oct 2016]. Disponible à l'adresse : http://www.phac-aspc.gc.ca/naciccni/flu-2016-grippe-fra.php.

7. Une déclaration d'un comité consultatif (DCC) Comité consultatif national de l'immunisation (CCNI). Addenda - Utilisation du VVAl chez les enfants et les adolescents. Chapitre sur la grippe du Guide canadien d'immunisation et Déclaration sur la vaccination antigrippale pour la saison 2016-2017 [Internet]. Ottawa: ASPC; 2016 [consulté le 12 oct 2016]. Disponible à l'adresse : http://www.phac-aspc. gc.ca/naci-ccni/flu-2016-grippe-addendum-children-enfantsfra.php.

8. Une déclaration d'un comité consultatif (DCC) Comité consultatif national de l'immunisation (CCNI). Addenda I'administration du WVI aux personnes allergiques aux œufs, Chapitre sur la grippe du Guide canadien d'immunisation et Déclaration sur la vaccination antigrippale pour la saison 2016-2017 [Internet]. Ottawa: ASPC; 2016 [consulté le 12 oct 2016]. Disponible à l'adresse : http://www.phac-aspc. gc.ca/naci-ccni/flu-2016-grippe-addendum-eggs-oeufs-fra. php.

9. Comité consultatif national de l'immunisation (CCNI). Guide canadien d'immunisation. Partie 4 - Agents d'immunisation active, Vaccin contre la varicelle [Internet]. Ottawa: ASPC; 
2016 [consulté le 12 oct 2016]. Disponible à l'adresse : http://healthycanadians.gc.ca/publications/healthy-livingvie-saine/4-canadian-immunization-guide-canadienimmunisation/index-fra.php?page=24.

10. Une déclaration d'un comité consultatif (DCC) Comité consultatif national de l'immunisation (CCNI). Mise à jour sur l'utilisation du vaccin conjugué 13-valent (Pneu-C-13) en plus du vaccin polysaccharidique 23-valent contre le pneumocoque (Pneu-P-23) chez les adultes immunocompétents âgés de 65 ans et plus Recommandation provisoire [Internet]. Ottawa: PHAC; 2016 [cited 2016 Nov 1]. Disponible à l'adresse : http://www. healthycanadians.gc.ca/publications/healthy-living-vie-saine/ update-pneu-c-13-and-pneu-p-23-mise-a-jour-2016/indexfra.php.

11. Une déclaration d'un comité consultatif (DCC) Comité consultatif national de l'immunisation (CCNI). Mise à jour des recommandations relatives à l'utilisation d'immunoglobulines antivaricelleuses (Varlg) pour la prévention de la varicelle chez les patients à risque [Internet]. Ottawa: ASPC; 2016 [consulté le 12 oct 2016]. Disponible à l'adresse : http:// healthycanadians.gc.ca/publications/healthy-living-viesaine/varicella-recommended-use-risk-patients-2016recommandations-varicelle-patients-risque/index-fra.php.

12. Une déclaration d'un comité consultatif (DCC) Comité consultatif national de l'immunisation (CCNI). Preuve d'immunité contre la varicelle - Mise à jour de 2015 [Internet]. Ottawa: ASPC; 2016 [consulté le 12 oct 2016]. Disponible à l'adresse : http://healthycanadians.gc.ca/ publications/healthy-living-vie-saine/varicella-proofimmunity-2015-varicelle-preuve-immunite/index-fra.php.

13. Agence de la santé publique du Canada [Internet]. Contactez-nous. [mise à jour le 11 août 2016; consulté le 24 oct 2016]. Disponible à l'adresse : http://www.phac-aspc. gc.ca/contac-fra.php.

14. Gouvernement du Canada [Internet]. Mise à jour du Guide canadien d'immunisation : Liste de diffusion. [mise à jour le 24 mai 2014; consulté le 24 oct 2016]. Disponible à l'adresse : http://healthycanadians.gc.ca/healthy-living-viesaine/immunization-immunisation/canadian-immunizationguide-canadien-immunisation/email-subscriptionabonnement-courriel-fra.php.

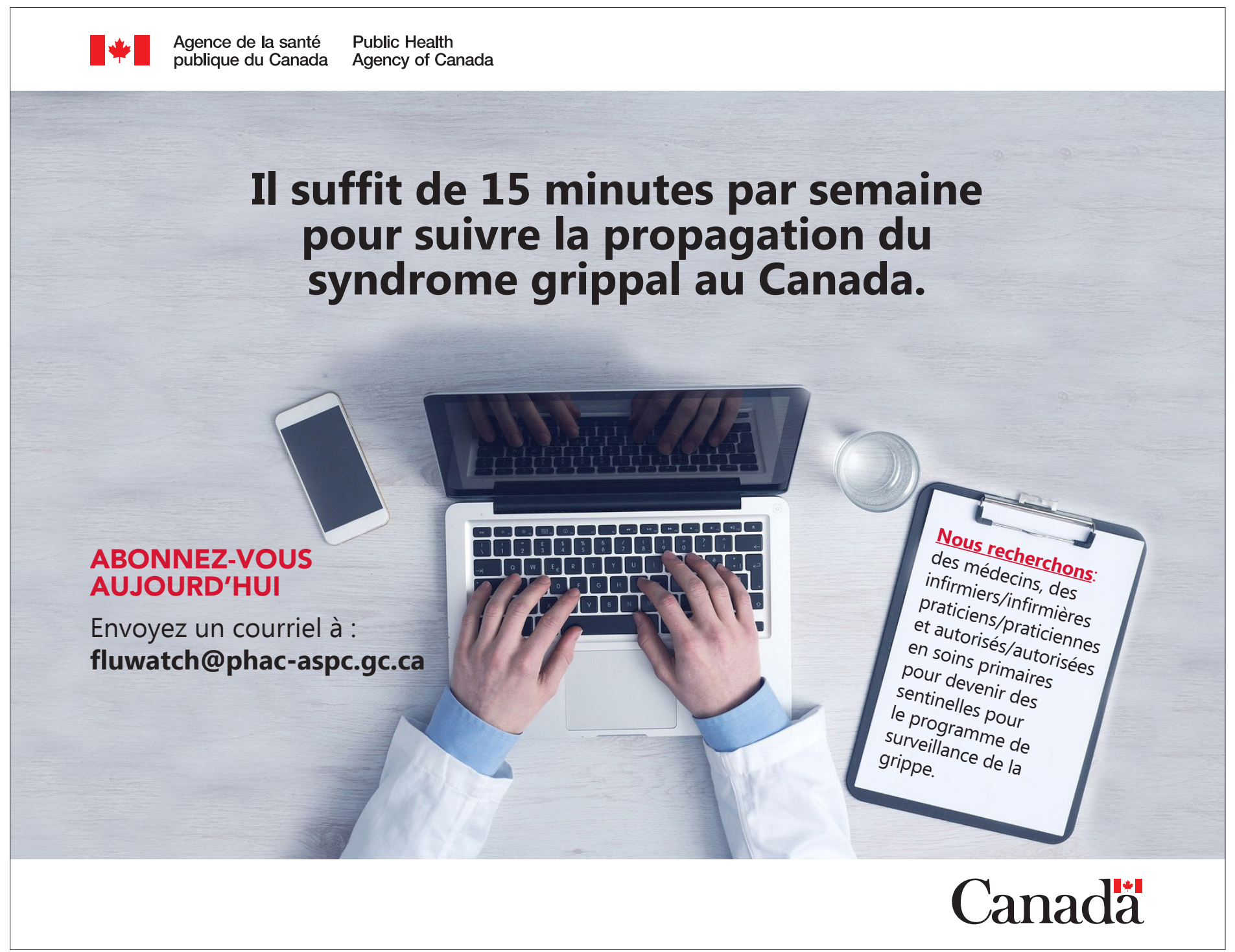

